\title{
Bessel Image Sharpness Analysis, Advances in Bessel Image Sharpness Analysis
}

\author{
Arjuna Pilippu Hewa Don ${ }^{1}$ and James peter ${ }^{2}$ \\ ${ }^{1}$ University of Manitoba \\ ${ }^{2}$ Department of Electrical and Computer Engineering, University of Manitoba 75A \\ Chancellor's Circle, Winnipeg, MB R3T 5V6 CANADA and Department of Mathematics, \\ Adiyaman University, 02040 Adiyaman, Turkey
}

November 9, 2020

\begin{abstract}
This paper introduces advances in the use of Bessel functions (cylinder functions) in achieving optimal sharpness of shapes delineated by collections of holes (dark valley regions) and bright regions in different areas of a video frame. An image hole is an island of low voxel intensities surrounded by varying voxel intensity peaks. The basic approach is to identify dominant collections of lights (high voxel intensity peaks) and darks (low voxel intensity clusters) in video frames. A main finding in this paper is that surface objects (recorded in images) are sharper wherever there are preponderant contrasting differences between image lights and darks. These contrasting differences lead to the highly accurate detection of shape holes (dark valleys) that delineate surface shapes recorded in sequences of video frames). With the use of cylindrical Bessel functions, contrasting differences between peaks and valleys can be measured in terms of voxel intensities and voxel indices.
\end{abstract}

\section{Hosted file}

BessellmageSharpnessAnalysis.pdf available at https://authorea.com/users/374350/articles/ 491886-bessel-image-sharpness-analysis-advances-in-bessel-image-sharpness-analysis 